\title{
Approximaitly Quasi-Prime Submodules and Some Related Concepts
}

\section{Haibat K. Mohammadali ${ }^{1}$}

Department of Mathematics

College of Computer Science and Mathematics

University of Tikrit

dr.mohammadali2013@gmail.com

Recived : 10\2\2019 Revised : 17\3\2019

\author{
Ali Sh. Ajeel ${ }^{2}$ \\ Department of Mathematics
}

College of Computer Science and Mathematics

University of Tikrit

Ali.shebl@st.tu.edu.iq

Available online : $24 / 4 / 2019$

\begin{abstract}
:
"Let $R$ be a commutative ring with identity and $B$ is a left unitary $R$-module. A proper submodule $E$ of $B$ is called a quasi-prime submodule, if whenever $r s b \in E$, where $r, s \in R, b \in B$ implies that either $r b \in E$ or $s b \in E$ ". As a generalization of a quasi-prime submodules, in this paper we introduce the concept of approximaitly quasi-prime submodules, where a proper submodule $E$ of $B$ is an approximaitly quasi-prime submodule, if whenever $r s b \in E$, where $r, s \in R, b \in B$ implies that either $r b \in E+\operatorname{soc}(B)$ or $s b \in E+\operatorname{soc}(B)$, where $\operatorname{soc}(B)$ is the intersection of all essential submodules of $B$. Many basic properties, characterization and examples of this concept are given. Furthermore, we study the behavior of approximaitly quasi-prime submodules under $R$-homomorphisms. Finally, we introduced characterizations of approximaitly quasi-prime submodule in class of multiplication modules.
\end{abstract}

Keywords. Prime submodules, Quasi-prime submodules, Approximaitly prime submodules, Socle of submodules, Multiplication Modules, Approximaitly quasi-prime submodules. 


\section{Introduction}

A quasi-prime submodule was introduced and studied in 1999 by [1] as a generalization of a prime submodule, where a proper submodule $E$ of an $R$ module $B$ is called a prime, if whenever $r b \in E$, where $r \in R, b \in B$ implies that either $b \in E$ or $r \in\left[E:_{R} B\right]$ where $\left[E:_{R} B\right]=\{r \in R: r B \subseteq E\} \quad$ [3], Recently several generalizations of quasi-prime submodules were introduced such as "Weakly quasiprime, Nearly quasi-prime, WE-quasi-prime, Weakly quasi 2-absorbing, Nearly quasi 2-absorbing, and Pseudo quasi 2-absorbing submodules see $[14,12,6,7,8,9]$ ". In this paper, we give another generalization of a quasi-prime submodule, where a proper submodule $E$ of $B$ is an approximaitly quasiprime submodule, if whenever $r s b \in E$, where $r, s \in R, b \in B$ implies that either $r b \in E+\operatorname{soc}(B)$ or $s b \in E+\operatorname{soc}(B)$. The concept of approximaitly quasi-prime submodule is also, generalization of the concept of approximaitly prime submodules which appear in [10], also generalization of prime submodules. Recall that an $R$-module $B$ is multiplication if every submodule $E$ of $B$ is of the form $E=I B$ for some ideal $I$ of $R$, in particular $E=\left[E:_{R} B\right] B$ [4]. Let $E$ and $D$ be a submodule of a multiplication $R$-module $B$ with $E=I B$ and $D=J B$ for some ideals $I$ and $J$ of $R$, then $E D=I J B$ that is $E D=I D$. In particular $E B=I B B=I B=E$. Also for any $b \in B$, we define $E b=E\langle b\rangle=I b[15]$.

\section{Basic properties of Approximaitly Quasi-Prime Submodules}

In this part of the paper we introduce the definition of approximaitly quasi-prime submodule, and give some basic properties, examples and characterizations of this concept.

Definition 2.1 A proper submodule $E$ of $B$ is said to be an approximaitly quasi-prime submodule(for short app-quasi-prime), if whenever $r s b \in E$, where $r, s \in R, b \in B$ implies that either $r b \in E+\operatorname{soc}(B)$ or $s b \in E+\operatorname{soc}(B)$. An ideal $I$ of a ring $R$ is an app- quasi-prime ideal of $R$ if and only if $I$ is an appquasi-prime submodule of an $R$-module $R$.

Remark 2.2 It is clear that every quasi-prime submodule is an app-quasi-prime, but the convers is not true in general, the following example explain that:

Example 2.3 Let $B=Z_{12}, R=Z$ and $E=\langle\overline{0}\rangle$, and $\operatorname{soc}\left(Z_{12}\right)=\langle\overline{2}\rangle . E$ is an app-quasi-prime submodule of $B$ since if $r s b \in E$, where $r, s \in Z, b \in Z_{12}$, implies that either $r b \in E+\langle\overline{2}\rangle=\langle\overline{2}\rangle$ or $s b \in E+$ $\langle\overline{2}\rangle=\langle\overline{2}\rangle$. But $E$ is not a quasi-prime submodule of $B$, since $3.4 . \overline{2} \in E$, but neither $3 . \overline{2} \in E$ nor $4 . \overline{2} \in E$.

The following proposition gives a characterization of app-quasi-prime submodules.

Proposition 2.4 Let $B$ be an $R$-module, and $E$ be a proper submodule of $B$. Then $E$ is an app-quasiprime submodule of $B$ if and only if whenever $I J D \subseteq E$, where $I, J$ are ideals in $R$ and $D$ is a submodule of $B$, implies that either $I D \subseteq E+$ $\operatorname{soc}(B)$ or $J D \subseteq E+\operatorname{soc}(B)$.

Proof $(\Longrightarrow)$ Suppose that $I J D \subseteq E$, where $I, J$ are ideals in $R$ and $D$ is a submodule of $B$, and with $I D \nsubseteq E+\operatorname{soc}(B)$ and $J D \nsubseteq E+\operatorname{soc}(B)$. So there exists $d_{1}, d_{2} \in D$ and $r \in I, s \in J$ such that $r d_{1} \notin$ $E+\operatorname{soc}(B)$ ands $d_{2} \notin E+\operatorname{soc}(B)$. Since $E$ is an app-quasi-prime submodule of $B$ and $r s d_{1} \in E$ and $r d_{1} \notin E+\operatorname{soc}(B)$ implies that $s d_{1} \in E+\operatorname{soc}(B)$. Also $r s d_{2} \in E$ and $s d_{2} \notin E+\operatorname{soc}(B)$ implies that $r d_{2} \in E+\operatorname{soc}(B)$. It follows that either $I D \subseteq E+$ $\operatorname{soc}(B)$ or $J D \subseteq E+\operatorname{soc}(B)$.

$(\Longleftarrow)$ Assume that $r s b \in E$, where $r, s \in R, b \in B$ implies that $(r)(s)(b) \subseteq E$, so by hypothesis either $(r)(b) \subseteq E+\operatorname{soc}(B)$ or $(s)(b) \subseteq E+\operatorname{soc}(B)$. Thus either $r b \in E+\operatorname{soc}(B)$ or $s b \in E+\operatorname{soc}(B)$. Hence $E$ is an app-quasi-prime submodule of $B$. 
As direct application of proposition 2.4 , we get the following corollaries.

Corollary 2.5 Let $B$ be an $R$-module, and $E$ be a proper submodule of $B$. Then $E$ is an app-quasiprime submodule of $B$ if and only if whenever $r s D \subseteq E$, where $r, s \in R$ and $D$ is a submodule of $B$, implies that either $r D \subseteq E+\operatorname{soc}(B)$ or $s D \subseteq E+$ $\operatorname{soc}(B)$.

Corollary 2.6 Let $B$ be an $R$-module, and $E$ be a proper submodule of $B$. Then $E$ is an app-quasiprime submodule of $B$ if and only if whenever $r I b \subseteq E$, where $r \in R, b \in B$ and $I$ is a ideal of $R$, implies that either $r b \in E+\operatorname{soc}(B)$ or $I b \subseteq E+$ $\operatorname{soc}(B)$.

Corollary 2.7 Let $B$ be an $R$-module, and $E$ be a proper submodule of $B$. Then $E$ is an app-quasiprime submodule of $B$ if and only if whenever $I J b \subseteq E$, where $I, J$ are ideals in $R$, and $b \in B$, implies that either $I b \subseteq E+\operatorname{soc}(B)$ or $J b \subseteq E+$ $\operatorname{soc}(B)$.

Proposition 2.8 Let $B$ be an $R$-module, and $E$ be a proper submodule of $B$ with $\operatorname{soc}(B) \subseteq E$. Then $E$ is an app-quasi-prime submodule of $B$ if and only if $[E+\operatorname{soc}(B): b]$ is a prime ideal of $R$ for each $b \in B$.

Proof $\Longrightarrow$ Let $r s \in[E+\operatorname{soc}(B): b]$, where $r, s \in R$, implies that $r s b \in E+\operatorname{soc}(B)$. But $\operatorname{soc}(B) \subseteq E$, it follows that $E+\operatorname{soc}(B)=E$, hence $r s b \in E$. But $E$ is an app-quasi-prime submodule of $B$, implies that either $r b \in E+\operatorname{soc}(B)$ or $s b \in E+$ $\operatorname{soc}(B)$. Thus either $r \in[E+\operatorname{soc}(B): b]$ or $s \in[E+$ $\operatorname{soc}(B): b]$.

( $\Longleftarrow$ ) Suppose that $r s b \in E$, where $r, s \in R, b \in B$ implies that either $r b s \in E \subseteq E+\operatorname{soc}(B)$, it follows that $r b s \in E+\operatorname{soc}(B)$, hence $r s \in[E+\operatorname{soc}(B): b]$. But $[E+\operatorname{soc}(B): b]$ is a prime ideal of $R$, implies that either $r \in[E+\operatorname{soc}(B): b] \quad$ or $\quad s \in[E+$
$\operatorname{soc}(B): b]$, it follows that either $r b \in E+\operatorname{soc}(B)$ or $s b \in E+\operatorname{soc}(B)$.

Proposition 2.9 Let $B$ be an $R$-module, and $E$ be a proper submodule of $B$. Then $E$ is an app-quasiprime submodule of $B$ if and only if $\left[E:_{B} r s\right] \subsetneq[E+$ $\left.\operatorname{soc}(B):_{B} r\right] \cup\left[E+\operatorname{soc}(B):_{B} s\right]$ for all $r, s \in R$.

Proof $(\Longrightarrow)$ Let $b \in\left[E:_{B} r s\right]$, implies that $r s b \in E$.

But $E$ is an app-quasi-prime submodule of $B$, then either $r b \in E+\operatorname{soc}(B)$ or $s b \in E+\operatorname{soc}(B)$. It follows that either $b \in\left[E+\operatorname{soc}(B):_{B} r\right]$ or $b \in$ $\left[E+\operatorname{soc}(B):_{B} s\right] . \quad$ Thus $\quad\left[E:_{B} r s\right] \subsetneq[E+$ $\left.\operatorname{soc}(B):_{B} r\right] \cup\left[E+\operatorname{soc}(B):_{B} s\right]$

$(\Longleftarrow)$ Now, let $r s b \in E$, where $r, s \in R, b \in B$, then $b \in\left[E:_{B} r s\right] \subsetneq\left[E+\operatorname{soc}(B):_{B} r\right] \cup[E+$ $\left.\operatorname{soc}(B):_{B} S\right]$, implies that $b \in\left[E+\operatorname{soc}(B):_{B} r\right]$ or $b \in\left[E+\operatorname{soc}(B):_{B} s\right]$. Hence $r b \in E+\operatorname{soc}(B)$ or $s b \in E+\operatorname{soc}(B)$. Thus $E$ is an app-quasi-prime submodule of $B$.

Proposition 2.10 Let $B$ be an $R$-module, and $E$ be a proper submodule of $B$ such that $E$ is an app-quasiprime submodule of $B$. Then $\left[E:_{R} r s b\right] \subsetneq[E+$ $\left.\operatorname{Soc}(B):_{R} r b\right] \cup\left[E+\operatorname{soc}(B):_{R} s b\right]$ for all $r, s \in R$, $b \in B$.

Proof Let $x \in\left[E:_{R} r s b\right]$, where $r, s \in R, b \in B$, implies that $r s(x b) \in E$. But $E$ is an app-quasi-prime submodule of $B$, then either $r(x b) \in E+\operatorname{soc}(B)$ or $s(x b) \in E+\operatorname{soc}(B)$, it follows that either $x \in[E+$ $\left.\operatorname{soc}(B):_{R} r b\right]$ or $x \in\left[E+\operatorname{soc}(B):_{R} s b\right]$. Hence $x \in\left[E+\operatorname{soc}(B):_{R} r b\right] \cup\left[E+\operatorname{soc}(B):_{R} s b\right]$. Thus $\left[E:_{R} r s b\right] \subsetneq\left[E+\operatorname{soc}(B):_{R} r b\right] \cup\left[E+\operatorname{soc}(B):_{R} s b\right]$.

Remark 2.11 Let $B$ be an $R$-module, and $E$ is an app-quasi-prime submodule of $B$, it is not necessary that $[E: B]$ is an app-quasi-prime ideal of $R$. For example in a $Z$-module $Z_{12},\langle\overline{0}\rangle$ is an app-quasiprime submodule, but $\left[\langle\overline{0}\rangle: Z_{12}\right]=12 Z$ is not appquasi-prime ideal of $Z$-module $Z$. Since $2.2 .3 \in 12 Z$, but $2.3 \notin 12 Z+\operatorname{soc}(Z)=12 Z+0=12 Z$. 
Proposition 2.12 Let $B$ be an $R$-module, and $E$ is an app-quasi-prime submodule of $B$ with $\operatorname{soc}(B) \subseteq$ $E$. Then $[E: B]$ is an app-quasi-prime ideal of $R$.

Proof Let $r s t \in[E: B]$, where $r, s, t \in R$, implies that $r s(t B) \subseteq E$. Thus since $E$ is an app-quasi-prime submodule of $B$, then by Corollary 2.5 either $r(t B) \subseteq E+\operatorname{soc}(B)$ or $s(t B) \subseteq E+\operatorname{soc}(B)$. But $\operatorname{soc}(B) \subseteq E$, implies that $E+\operatorname{soc}(B)=E$. Hence either $r(t B) \subseteq E$ or $s(t B) \subseteq E$. That is either $r t \in\{E: B] \subseteq[E: B]+\operatorname{soc}(R) \quad$ or $\quad s t \in\{E: B] \subseteq$ $[E: B]+\operatorname{soc}(R)$. Therefore $[E: B]$ is an app-quasiprime ideal of $R$.

Recall that an $R$-module $B$ is faithful if $a n n_{R} B=(0)$ [4].

Before we introduce the converse of Proposition 2.12 we recall the following lemmas:

Lemma 2.13 [4, Coro. 2.14] Let $B$ be a faithful multiplication $R$-module then $\operatorname{soc}(R) B=\operatorname{soc}(B)$.

Recall that an $R$-module $B$ is a non-singular provided that $Z(B)=B$, where $Z(B)=\{b \in B$ : $b I=0$ for some essential ideal $I$ of $R\}$ [5].

Lemma 2.14 [5, Coro. 1.26] If $B$ is a nonsingular $R$-module, then $\operatorname{soc}(R) B=\operatorname{soc}(B)$.

Proposition 2.15 Let $B$ be a faithful multiplication $R$-module and $E$ is a proper submodule of $B$. If $[E: B]$ is an app-quasi-prime ideal of $R$, then $E$ is an app-quasi-prime submodule of $B$.

Proof Suppose that $r s b \in E$, where $r, s \in R, b \in B$ implies that $r s(b) \subseteq E$. Since $B$ be a multiplication, then $(b)=J B$ for some ideal $J$ of $R$. That is $r s J B \subseteq$ $E$, it follows that $r S J \subseteq[E: B]$. But $[E: B]$ is an appquasi-prime ideal of $R$, then by Corollary 2.5 either $r J \subseteq[E: B]+\operatorname{soc}(R)$ or $s J \subseteq[E: B]+\operatorname{soc}(R)$, it follows that either $r J B \subseteq[E: B] B+\operatorname{soc}(R) B$ or $s J B \subseteq[E: B] B+\operatorname{soc}(R) B$. Hence, by Lemma 2.13
$\operatorname{soc}(R) B=\operatorname{soc}(B)$, and since $B$ is multiplication $[E: B] B=E$. therefore either $r b \in E+\operatorname{soc}(B)$ or $s b \in E+\operatorname{soc}(B)$. Hence $E$ is an app-quasi-prime submodule of $B$.

Proposition 2.16 Let $B$ be a non-singular multiplication $R$-module and $E$ is a proper submodule of $B$. If $[E: B]$ is an app-quasi-prime ideal of $R$, then $E$ is an app-quasi-prime submodule of $B$.

Proof Follows in similar way of Proposition 2.15 and using Lemma 2.14 .

Lemma 2.17 [13, Coro. Of Theo. 9] Let $I$ and $J$ be ideals of a ring $R$, and $B$ is a finitely generated multiplication $R$-module. Then $I B \subseteq J B$ if and only if $I \subseteq J+a n n B$.

Proposition 2.18 Let $B$ be a faithful finitely generated multiplication $R$-module. If $J$ is an appquasi-prime ideal of $R$, then $J B$ is an app-quasi-prime submodule of $B$.

Proof Suppose that $r s b \in J B$, where $r, s \in R, b \in B$. Then $r s(b) \subseteq J B$. Since $B$ is a multiplication, implies that $(b)=I B$ for some ideal $I$ of $R$. Thus $r s I B \subseteq J B$. But $B$ is a finitely generated, so by Lemma 2.17 $r s I \subseteq J+a n n B$. But $B$ is faithful, it follows that $a n n B=(0)$, hence $r s I \subseteq J$, but $J$ is an app-quasiprime ideal of $R$, then by Corollary 2.5 either $r I \subseteq J+\operatorname{soc}(R)$ or $s I \subseteq J+\operatorname{soc}(R)$, it follows that either $\quad r I B \subseteq J B+\operatorname{soc}(R) B \quad$ or $\quad s I B \subseteq J B+$ $\operatorname{soc}(R) B$. But by Lemma $2.13 \operatorname{soc}(R) B=\operatorname{sov}(B)$. Hence $r I B \subseteq J B+\operatorname{soc}(B)$ or $s I B \subseteq J B+\operatorname{soc}(B)$, it follows that either $r b \in J B+\operatorname{soc}(B)$ or $s b \in J B+$ $\operatorname{soc}(B)$. Therefor $J B$ is an app-quasi-prime submodule of $B$.

Proposition 2.19 Let $B$ be a finitely generated multiplication non-singular $R$-module, and $J$ is an app-quasi-prime ideal of $R$ with $a n n B \subseteq J$. Then $J B$ is an app-quasi-prime submodule of $B$. 
Proof Similar steps of Proposition 2.18 and using Lemma 2.14 and the condition $a n n B \subseteq J$ implies that $J+\operatorname{ann} B=J$.

Remark 2.20 The intersection of any two appquasi-prime submodules of an $R$-module $B$ not necessary app-quasi-prime submodule of $B$, the following example shows that.

Example 2.21 Let $B$ be the $Z$-module $Z$ and $E=2 Z$ and $D=3 Z$. It is clear that $E$ and $D$ are app-quasiprime submodules of $B$, but $E \cap D=6 Z$ is not appquasi-ptime submodule of $B$ since 2.3.1 $\in Z$, but $2.1 \notin 6 Z+\operatorname{soc}(B)$ and $3.1 \notin 6 Z+\operatorname{soc}(B)$, where $\operatorname{soc}(B)=(0)$.

Proposition 2.22 Let $B$ be an $R$-module, and $E, D$ are app-quasi-prime submodules with $E \subseteq \operatorname{soc}(B)$ and $D \subseteq \operatorname{soc}(B)$. Then $E \cap D$ is an app-quasi-prime submodule of $B$.

Proof Suppose that $r s b \in E \cap D$, where $r, s \in R$, $b \in B$, then $r s b \in E$ and $r s b \in D$. Since both $E$ and $D$ are app-quasi-prime submodules of $B$, so either $r b \in E+\operatorname{soc}(B)$ or $s b \in E+\operatorname{soc}(B)$ and either $r b \in D+\operatorname{soc}(B)$ or $s b \in D+\operatorname{soc}(B)$. But $E \subseteq$ $\operatorname{soc}(B)$ and $D \subseteq \operatorname{soc}(B)$, it follows that $E+$ $\operatorname{soc}(B)=\operatorname{soc}(B)$ and $D+\operatorname{soc}(B)=\operatorname{soc}(B)$ and $E \cap D \subseteq \operatorname{soc}(B)$, implies that $E \cap D+\operatorname{soc}(B)=$ $\operatorname{soc}(B)$. Thus we have either $r b \in E \cap D+\operatorname{soc}(B)$ or $s b \in E \cap D+\operatorname{soc}(B)$. That is $E \cap D$ is an appquasi-prime submodule of $B$.

Lemma 2.23 [11, Lemma 2.3.15] Let $B$ be an $R$-module, and $E, D, F$ are submodules of $B$ with $D$ is contained in $F$ then $(E+D) \cap F=(E \cap F)+$ $(D \cap F)=(E \cap F)+D$.

Lemma 2.24 [2, Coro. 9.9] Let $B$ be an $R$ module, and $E$ submodule of $B$, then $\operatorname{soc}(E)=E \cap$ $\operatorname{soc}(B)$.

Proposition 2.25 Let $B$ be an $R$-module, and $E, D$ are two submodules of $B$ with $D$ is not contained in $E$ and $\operatorname{soc}(B) \subseteq D$. If $E$ is an app-quasi-prime submodule of $B$, then $E \cap D$ is an app-quasi-prime submodule of $D$.

Proof Since $D$ is not contained in $E$, then $E \cap D$ is a proper submodule of $D$. Now, let $r s b \in E \cap D$ where $r, s \in R, \quad b \in D \subseteq B$, then $r s b \in E$ and $r s b \in D$. But $E$ is an app-quasi-prime submodule of $B$, then either $r b \in E+\operatorname{soc}(B)$ or $s b \in E+$ $\operatorname{soc}(B)$, since $b \in D$, it follows that either $r b \in(E+$ $\operatorname{soc}(B)) \cap D$ or $\quad s b \in(E+\operatorname{soc}(B)) \cap D$. Since $\operatorname{soc}(B) \subseteq D$, then by Lemma 2.23

We have $(E+\operatorname{soc}(B)) \cap D=(E \cap D)+\operatorname{soc}(B) \cap$ $D$, and by Lemma 2.24 we have $\operatorname{soc}(B) \cap D=$ $\operatorname{soc}(D)$. Hence either $r b \in E \cap D+\operatorname{soc}(D)$ or $s b \in E \cap D+\operatorname{soc}(D)$. Thus $E \cap D$ is an app-quasiprime submodule of $D$.

Proposition 2.26 Let $f \in \operatorname{Hom}\left(B, B^{\prime}\right)$ be an $R$ epimorphism, and $E$ is an app-quasi-prime submodule of $B^{\prime}$. Then $f^{-1}(E)$ is an app-quasi-prime submodule of $B$.

Proof It is clear that $f^{-1}(E)$ is a proper submodule of $B$. Now, let $r s b \in f^{-1}(E)$, where $r, s \in R, b \in B$, implies that $r s f(b) \in E$. since $E$ is an app-quasiprime submodule of $B^{\prime}$, so either $r f(b) \in E+$ $\operatorname{soc}\left(B^{\prime}\right)$ or $s f(b) \in E+\operatorname{soc}\left(B^{\prime}\right)$. Hence either $r b \in f^{-1}(E)+f^{-1}\left(\operatorname{soc}\left(B^{\prime}\right)\right) \subseteq f^{-1}(E)+\operatorname{soc}(B)$ or $\quad s b \in f^{-1}(E)+f^{-1}\left(\operatorname{soc}\left(B^{\prime}\right)\right) \subseteq f^{-1}(E)+$ $\operatorname{soc}(B)$. That is either $r b \in f^{-1}(E)+\operatorname{soc}(B)$ or $s b \in f^{-1}(E)+\operatorname{soc}(B)$. Therefore $f^{-1}(E)$ is an appquasi-prime submodule of $B$.

Proposition 2.27 Let $f \in \operatorname{Hom}\left(B, B^{\prime}\right)$ be an $R$ epimorphism, and $E$ be an app-quasi-prime submodule of $B$ with $\operatorname{Ker} f \subseteq E$. Then $f(E)$ is an app-quasi-prime submodule of $B^{\prime}$.

Proof $f(E)$ is a proper submodule of $B^{\prime}$. If not, suppose that $f(E)=B^{\prime}$, let $b \in B$, then $f(b) \in B^{\prime}=$ $f(E)$, implies that $f(b)=f(e)$ for some $e \in E$, it follows tat $f(b-e)=0$, so $b-e \in \operatorname{Ker} f \subseteq E$, hence $b \in E$, that is $E=B$ contradiction. Now let 
$r s b^{\prime} \in f(E)$, where $r, s \in R, b^{\prime} \in B^{\prime}$. Since $f$ is an epimorphism, then $f(b)=b^{\prime}$ for some $b \in B$, thus $\operatorname{rsf}(b) \in f(E)$, it follows that $\operatorname{rsf}(b)=f(e)$ for some $e \in E$. That is $f(r s b-e)=0$, so $r s b-e \in$ $\operatorname{Ker} f \subseteq E$, implies that $r s b \in E$. But $E$ is an appquasi-prime submodule of $B$, then either $r b \in E+$ $\operatorname{soc}(B)$ or $s b \in E+\operatorname{soc}(B)$, it follows that either $r f(b) \in f(E)+f(\operatorname{soc}(B)) \subseteq f(E)+\operatorname{soc}\left(B^{\prime}\right)$ or $s f(b) \in f(E)+f(\operatorname{soc}(B)) \subseteq f(E)+\operatorname{soc}\left(B^{\prime}\right)$.

Hence either $r b^{\prime} \in f(E)+\operatorname{soc}\left(B^{\prime}\right)$ or $s b^{\prime} \in f(E)+$ $\operatorname{soc}\left(B^{\prime}\right)$. Thus $f(E)$ is an app-quasi-prime submodule of $B^{\prime}$.

Proposition 2.28 Let $B, B^{\prime}$ be $R$-modules, and $E$ be a proper submodule of $B^{\prime}$, such that $E+$ $\operatorname{soc}\left(B^{\prime}\right)$ is a quasi-prime submodule of $B^{\prime}$, with $\operatorname{Hom}_{R}\left(B, E+\operatorname{soc}\left(B^{\prime}\right)\right)$ is a proper submodule of $\operatorname{Hom}_{R}\left(B, B^{\prime}\right)$. Then $\operatorname{Hom}_{R}\left(B, E+\operatorname{soc}\left(B^{\prime}\right)\right)$ is appquasi-prime submodule of $\operatorname{Hom}_{R}\left(B, B^{\prime}\right)$.

Proof Suppose that $r s f \in \operatorname{Hom}_{R}\left(B, E+\operatorname{soc}\left(B^{\prime}\right)\right)$ where $r, s \in R, f \in \operatorname{Hom}_{R}\left(B, B^{\prime}\right)$. Then for each $b \in B$, we have $r s f(b) \in E+\operatorname{soc}\left(B^{\prime}\right)$. But $E+$ $\operatorname{soc}\left(B^{\prime}\right)$ is quasi-prime submodule of $B^{\prime}$, then either $r f(b) \in E+\operatorname{soc}\left(B^{\prime}\right)$ of $s f(b) \in E+\operatorname{soc}\left(B^{\prime}\right)$. That is $\quad r f \in \operatorname{Hom}_{R}\left(B, E+\operatorname{soc}\left(B^{\prime}\right)\right) \subseteq \operatorname{Hom}_{R}(B, E+$ $\left.\operatorname{soc}\left(B^{\prime}\right)\right)+\operatorname{soc}\left(\operatorname{Hom}_{R}\left(B, B^{\prime}\right)\right)$ or $s f \in \operatorname{Hom}_{R}(B$, $\left.E+\operatorname{soc}\left(B^{\prime}\right)\right) \subseteq \operatorname{Hom}_{R}\left(B, E+\operatorname{soc}\left(B^{\prime}\right)\right)+$ $\operatorname{soc}\left(\operatorname{Hom}_{R}\left(B, B^{\prime}\right)\right)$. Thus $\operatorname{Hom}_{R}\left(B, E+\operatorname{soc}\left(B^{\prime}\right)\right.$ is app-quasi-prime submodule of $\operatorname{Hom}_{R}\left(B, B^{\prime}\right)$.

Proposition 2.29 Let $B=B_{1} \oplus B_{2}$ be an $R$ module, where $B_{1}, B_{2}$ be modules, and $E=E_{1} \oplus E_{2}$ be submodule of $B$ with $E_{1}, E_{2}$ are submodules of $B_{1}, B_{2}$ respectively with $E \subseteq \operatorname{soc}(B)$. If $E$ is an appquasi-prime submodule of $B$, then $E_{1}$ and $E_{2}$ are appquasi-prime submodules of $B_{1}$ and $B_{2}$ respectively.

Proof Suppose that $b_{1} \in E_{1}$, where $r, s \in R, b_{1} \in$ $B_{1}$, then $r s\left(b_{1}, 0\right) \in E$. Since $E$ is an app-quasiprime submodule of $B$, then either $r\left(b_{1}, 0\right) \in E+$ $\operatorname{soc}(B)$ or $s\left(b_{1}, 0\right) \in E+\operatorname{soc}(B)$. But $E \subseteq \operatorname{soc}(B)$, implies that $E+\operatorname{soc}(B)=\operatorname{soc}(B)=\operatorname{soc}\left(B_{1}\right) \oplus$
$\operatorname{soc}\left(B_{2}\right)$. If $r\left(b_{1}, 0\right) \in \operatorname{soc}\left(B_{1}\right) \oplus \operatorname{soc}\left(B_{2}\right)$, implies that $r b_{1} \in \operatorname{soc}\left(B_{1}\right) \subseteq E_{1}+\operatorname{soc}\left(B_{1}\right)$ If $s\left(b_{1}, 0\right) \in$ $\operatorname{soc}\left(B_{1}\right) \oplus \operatorname{soc}\left(B_{2}\right)$, implies that $s b_{1} \in \operatorname{soc}\left(B_{1}\right) \subseteq$ $E_{1}+\operatorname{soc}\left(B_{1}\right)$. Hence $r b_{1} \in E_{1}+\operatorname{soc}\left(B_{1}\right)$ or $s b_{1} \in$ $E_{1}+\operatorname{soc}\left(B_{1}\right)$. Therefore $E_{1}$ is an app-quasi-prime submodule of $B_{1}$

Similarly $E_{2}$ is an app-quasi-prime submodule of $B_{2}$.

Proposition 2.30 Let $B=B_{1} \oplus B_{2}$ be an $R$ module, where $B_{1}, B_{2}$ is an $R$-modules, then.

1) $E$ is an app-quasi-prime submodule of $B_{1}$, with $E \subseteq \operatorname{soc}\left(B_{1}\right)$ and $B_{2}$ is a semi simple if and only if $E \oplus B_{2}$ is an app-quasi-prime submodule of $B$.

2) $D$ is an app-quasi-prime submodule of $B_{2}$ with $D \subseteq \operatorname{soc}\left(B_{2}\right)$ and $B_{1}$ is a semi simple if and only if $B_{1} \oplus D$ is an app-quasi-prime submodule of $B$.

\section{Proof}

(1) $(\Longrightarrow)$ Let $r s\left(b_{1}, b_{2}\right) \in E \oplus B_{2}$, where $r, s \in R$, $\left(b_{1}, b_{2}\right) \in B$, then $r s b_{1} \in E$ and $r s b_{2} \in B_{2}$. But $E$ is an app-quasi-prime submodule of $B_{1}$, and $E \subseteq$ $\operatorname{soc}\left(B_{1}\right)$, then either $r b_{1} \in E+\operatorname{soc}\left(B_{1}\right)=\operatorname{soc}\left(B_{1}\right)$ or $s b_{1} \in E+\operatorname{soc}\left(B_{1}\right)=\operatorname{soc}\left(B_{1}\right)$. Now since $B_{2}$ is a semi simple, then by $[2, \mathrm{p} 221] \operatorname{soc}\left(B_{2}\right)=B_{2}$. So, if $r b_{1} \in E+\operatorname{soc}\left(B_{1}\right)=\operatorname{soc}\left(B_{1}\right) \quad$ then $\quad\left(b_{1}, b_{2}\right) \in$ $\operatorname{soc}\left(B_{1}\right) \oplus \operatorname{soc}\left(B_{2}\right) \quad=\operatorname{soc}\left(B_{1} \oplus B_{2}\right) \subseteq E \oplus B_{2}+$ $\operatorname{soc}\left(B_{1} \oplus B_{2}\right)$. If if $s b_{1} \in E+\operatorname{soc}\left(B_{1}\right)=\operatorname{soc}\left(B_{1}\right)$ then $\quad s\left(b_{1}, b_{2}\right) \in \operatorname{soc}\left(B_{1}\right) \oplus \operatorname{soc}\left(B_{2}\right)=\operatorname{soc}\left(B_{1} \oplus\right.$ $\left.B_{2}\right) \subseteq E \oplus B_{2}+\operatorname{soc}\left(B_{1} \oplus B_{2}\right)$. Thus $E \oplus B_{2}$ is an app-quasi-prime submodule of $B$.

$(\Longleftarrow)$ Let $b_{1} \in E$, where $r, s \in R, b_{1} \in B_{1}$, then for each $b_{2} \in B_{2} r s\left(b_{1}, b_{2}\right) \in E \oplus B_{2}$. But $E \oplus B_{2}$ is an app-quasi-prime submodule of $B$, so, either $r\left(b_{1}, b_{2}\right) \in E \oplus B_{2}+\operatorname{soc}\left(B_{1} \oplus B_{2}\right) \quad$ or $\quad s\left(b_{1}, b_{2}\right) \in$ $E \oplus B_{2} \quad+\operatorname{soc}\left(B_{1} \oplus B_{2}\right)$. If $\quad r\left(b_{1}, b_{2}\right) \in E \oplus B_{2}+$ $\operatorname{soc}\left(B_{1}\right) \oplus \operatorname{soc}\left(B_{2}\right), \quad$ since $E \subseteq \operatorname{soc}\left(B_{1}\right)$, then $E+\operatorname{soc}\left(B_{1}\right)=\operatorname{soc}\left(B_{1}\right), \quad$ and $\operatorname{soc}\left(B_{2}\right)=B_{2} \quad$ so, $r\left(b_{1}, b_{2}\right) \in E \oplus B_{2}+\left(E+\operatorname{soc}\left(B_{2}\right)\right) \oplus B_{2}$ implies that $r\left(b_{1}, b_{2}\right) \in\left(E+\operatorname{soc}\left(B_{2}\right)\right) \oplus B_{2}$, it follows that $r b_{1} \in E+\operatorname{soc}\left(B_{1}\right)$. Similarly if $s\left(b_{1}, b_{2}\right) \in E \oplus B_{2}+$ $\operatorname{soc}\left(B_{1}\right) \bigoplus \operatorname{soc}\left(B_{2}\right)$, implies that $s b_{1} \in E+\operatorname{soc}\left(B_{1}\right)$. Therefore $E$ is an app-quasi-prime submodule of $B_{1}$. 
(2) In similar way we can prove (2).

Remark 2.31 It is clear that every prime submodule is an app-quasi-prime submodule while the convers is not true in general as the following example shows that.

Example 2.32 Consider the $Z$-module $Z_{4}$, the submodule $E=\langle\overline{0}\rangle$ is an app-quasi-prime submodule of $Z_{4}$, since for each $r, s \in Z$, and $b \in Z_{4}$, with $r s b \in E$, we have either $r b \in E+\operatorname{soc}\left(Z_{4}\right)=E+$ $\langle\overline{2}\rangle$ or $s b \in E+\operatorname{soc}\left(Z_{4}\right)=E+\langle\overline{2}\rangle$. But $\langle\overline{0}\rangle$ is not prime submodule of $Z_{4}$, because $2 . \overline{2} \in\langle\overline{0}\rangle, 2 \in Z$, $\overline{2} \in Z_{4}$, but $\overline{2} \notin\langle\overline{0}\rangle$ and $2 \notin\left[\langle\overline{0}\rangle: Z_{4}\right]=4 Z$.

Recall that a proper submodule $E$ of an $R$ module $B$ is called an app-prime submodule of $B$, if whenever $r b \in E$, with $r \in R, b \in B$, implies that either $b \in E+\operatorname{soc}(B)$ or $r B \subseteq E+\operatorname{soc}(B)$ [10].

Remark 2.33 It is clear that every app-prime submodule is an app-quasi-prime submodule, while the converse is not true in general, as the following example shows that.

Example 2.34 Consider the $Z$-module $Z \oplus Z$, and $E=(0) \oplus 2 Z, E$ is not app-prime, since $2(0,1) \in E$, but $(0,1) \notin E+\operatorname{soc}(Z \oplus Z)$, and $2 \notin[(0) \oplus 2 Z+$ $\operatorname{soc}(Z \oplus Z): Z \oplus Z]=(0)$. But $E$ is an app-quasiprime because $E$ is a quasi-prime submodule of $Z \oplus Z$.

Proposition 2.35 Let $B$ be an $R$-module, and $E$ be a proper submodule of $B$, with $\operatorname{soc}(B) \subseteq E$. Then $E$ is an app-quasi-prime submodule of $B$ if and only if $\left[E:_{R} I\right]$ is an app-quasi-prime submodule of $B$ for every ideal $I$ of $R$.

Proof $(\Longrightarrow)$ Let $r s b \in\left[E:_{R} I\right]$, with $r, s \in R, b \in B$, implies that $r s b I \subseteq E$, that is $r s b a \in E$ for each $a \in I$. Since $E$ is an app-quasi-prime submodule of $B$, it follows that either $r b a \in E+\operatorname{soc}(B)$ or $\operatorname{sba} \in E+\operatorname{soc}(B)$, but $\operatorname{soc}(B) \subseteq E$, implies that
$E+\operatorname{soc}(B)=E$. Thus either $r b a \in E$ or $s b a \in E$. That is either $r b \in\left[E:_{R} I\right] \subseteq\left[E:_{R} I\right]+\operatorname{soc}(B)$ or $r b \in\left[E:_{R} I\right] \subseteq\left[E:_{R} I\right]+\operatorname{soc}(B)$. Hence $\left[E:_{R} I\right]$ is an app-quasi-prime submodule of $B$.

$(\Longleftarrow)$ Since $\left[E:_{R} I\right]$ is an app-quasi-prime submodule of $B$ for each ideal $I$ of $R$, thus put $I=R$, we get $[E: R]=E$ is an app-quasi-prime submodule of $B$.

Proposition 2.36 Let $B$ be a multiplication $R$ module, and $E$ be a proper submodule of $B$. Then $E$ is an app-quasi-prime submodule of $B$ if and only if whenever $F D b \subseteq E$, for some submodules $F$ and $D$ of $B$ and $b \in B$, then either $F b \subseteq E+\operatorname{soc}(B)$ or $D b \subseteq E+\operatorname{soc}(B)$.

Proof $(\Longrightarrow)$ Suppose that $F D b \subseteq E$, for some submodules $F$ and $D$ of $B$ and $b \in B$. But $B$ is a multiolication then $F=I B$ and $D=J B$ for some ideals $I, J$ of $R$, thus $F D b=I J b \subseteq E$. But $E$ is an app-quasi-prime submodule of $B$, then by Corollary 2.7 either $I b \subseteq E+\operatorname{soc}(B)$ or $J b \subseteq E+\operatorname{soc}(B)$. It follows that either $F b \subseteq E+\operatorname{soc}(B)$ or $D b \subseteq E+$ $\operatorname{soc}(B)$.

$(\Longleftarrow)$ Assume that $I J b \subseteq E$, where $I, J$ are ideals in $R$ and $b \in B$. Since $B$ is a multiplication it follows that, $I D b=F D b \subseteq E$, so by hypothesis either $D b \subseteq E+$ $\operatorname{soc}(B)$ or $F b \subseteq E+\operatorname{soc}(B)$, that is either $I b \subseteq E+$ $\operatorname{soc}(B)$ or $J b \subseteq E+\operatorname{soc}(B)$. Hence by Corollary 2.7 Then $E$ is an app-quasi-prime submodule of $B$.

Proposition 2.37 Let $B$ be a multiplication $R$ module, and $E$ be a proper submodule of $B$. Then $E$ is an app-quasi-prime submodule of $B$ if and only if whenever $F D L \subseteq E$, for some submodules $F, D$ and $L$ of $B$, then either $F L \subseteq E+\operatorname{soc}(B)$ or $D L \subseteq E+$ $\operatorname{soc}(B)$.

Proof $(\Longrightarrow)$ Suppose that $F D L \subseteq E$, for some submodules $F, D$ and $L$ of $B$. But $B$ is a multiolication then $F=I B$ and $D=J B$ for some ideals $I, J$ of $R$, thus $F D L=I J L \subseteq E$. Since $E$ is an 
app-quasi-prime submodule of $B$, then by Proposition 2.4 either $I L \subseteq E+\operatorname{soc}(B)$ or $J L \subseteq E+\operatorname{soc}(B)$. It follows that either $F L \subseteq E+\operatorname{soc}(B)$ or $D L \subseteq E+$ $\operatorname{soc}(B)$.

$(\Longleftarrow$ ) Assume that $I J L \subseteq E$, where $I, J$ are ideals in $R$ and $L$ is a submodule of $B$. Since $B$ is a multiplication it follows that, $I D L=F D L \subseteq E$, so by hypothesis either $F L \subseteq E+\operatorname{soc}(B)$ or $D L \subseteq E+\operatorname{soc}(B)$, that is either $I L \subseteq E+\operatorname{soc}(B)$ or $J L \subseteq E+\operatorname{soc}(B)$. Hence by Proposition 2.4 $E$ is an app-quasi-prime submodule of $B$.

Proposition 2.38 Let $B$ be a faithful finitely generated multiplication $R$-module, and $E$ be a proper submodule of $B$ with $\operatorname{soc}(B) \subseteq E$. then the following statements are equivalent.

1) $E$ is an app-quasi-prime submodule of $B$.

2) $\left[E:_{R} B\right]$ is an app-quasi-prime ideal of $R$.

3) $E=I B$ for some app-quasi-prime ideal $I$ of $R$.

Proof $(1) \Rightarrow(2)$ Follows by Proposition 2.12

(2) $\Longrightarrow(1)$ Follows by Proposition 2.15

$(2) \Longrightarrow(3)$ Since $\left[E:_{R} B\right]$ is an app-quasi-prime ideal of $R$, and $E=\left[E:_{R} B\right] B$, it is follows that $E=I B$ and $I=\left[E:_{R} B\right]$ an app-quasi-prime ideal of $R$.

(3) $\Longrightarrow$ (2) Suppose that $E=I B$ for some app-quasiprime ideal $I$ of $R$. But $B$ is a multiplication we have $E=\left[E:_{R} B\right] B=I B$. Thus since $B$ is faithful finitely generated multiplication, then by Lemma 2.17 we have $I=\left[E:_{R} B\right]$, it follows that $\left[E:_{R} B\right]$ is an appquasi-prime ideal of $R$.

\section{References}

[1] Abdul - Razak H.M . ; Quasi-Prime Modules and Quasi-Prime submodules; M.Sc. Thesis, University of Baghdad, 1999.

[2] Anderson, F.W. and Fuller, K.R. ; Rings and Categories of Modules; Springer-Verlag, New York. 1992.

[3] Dauns, J. ; Prime Modules; J. Reine Angew, Math. 2 (1978), 156-181.
[4] El-Bast, Z.A. and Smith, P.F. ; Multiplication modules; Comm. In Algebra 16(4)(1988), 755-779.

[5] Goodearl, K.R. ; Ring Theory, Non Singular Rings and Modules Marcel Dekker; Inc. New York and Basel,1976.

[6] Haibat K.M. and Saif A.H. ; WE-primary submodules and WE-quasi-prime submodules; Tikrit J. of pure sci. (2018), To Appear.

[7] Haibat K.M. and Khalaf H.A. ; Weakly-Quasi2Absorbing submodules; Tikrit J. of pure sci. 23(7)(2018), 101-104.

[8] Haibat K.M. and Khalaf H.A. ; Nearly Quasi2Absorbing submodules; Tikrit J. of pure sci. 23(9)(2018), 99-102.

[9] Haibat K.M. and Omer A.A. ; Pseudo Quasi2Absorbing submodules and Some Related concepts; Ibn AL-Haitham J. for Pure and Applied sci. rr(2)(2019), To Appear

[10] ] Haibat K.M. and Ali Sh.A. ; Approximaitly Prime submodules and Some Related concepts; Ibn AL-Haitham J. for Pure and Applied sci. 32(2)(2019), To Appear

[11] Kasch, F. ; Modules and Rings; London Math. Soc. Monographs, New York, Academic press, 1982. [12] Nuhad S.A. and Adwia J.A. ; Nearly Quasiprime submodules; Int. J. Addv. Res. 5(1)(2017), 170-180.

[13] Smith, P.F. ; Some Remarks On Multiplication Module; Arch. Math. So, (1988), 223-225.

[14] Waad, K.H. ; Weakly Quasi-prime Modules and Weakly Quasi-prime Submodules; M. Sc. Thesis, Tikrit university 2008.

[15] Yousefian A. and Soheilnia F. ; 2-Absorbing and Weakly 2-Absorbing submodules; THAI J. of Math. 3(9)(2011), 577-584. 


\title{
المقاسات الجزئية الاولية الظاهرية تقريباً ومفاهيم ذات علاقة
}

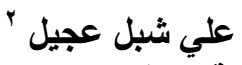

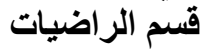 \\ كليه علوم الحاسوب والرياض \\ جامعة تكريت
}

\author{
هيبة كريم محمدعلي' \\ قسم الراضيات \\ كليه علوم الحاسوب والريات \\ جامعة تكريت
}

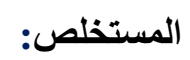

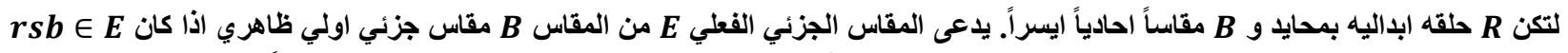
حيث

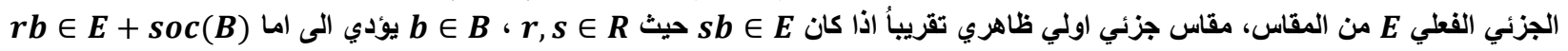
او soc(B) حو so

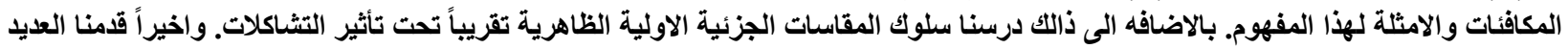

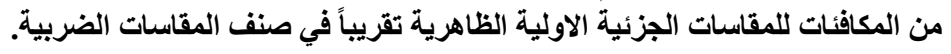

